OPEN ACCESS

Edited by:

Laurel J. Gershwin,

University of California, Davis,

United States

Reviewed by:

Martin Faldyna,

Veterinary Research Institute (VRI),

Czechia

Sabine Hammer,

University of Veterinary Medicine

Vienna, Austria

*Correspondence:

Hans Klingemann

Hans.Klingemann@ImmunityBio.com

Specialty section:

This article was submitted to Comparative Immunology, a section of the journal

Frontiers in Immunology

Received: 08 February 2021

Accepted: 16 July 2021

Published: 05 August 2021

Citation:

Klingemann H (2021)

Immunotherapy for Dogs:

Still Running Behind Humans.

Front. Immunol. 12:665784.

doi: 10.3389/fimmu.2021.665784

\section{Immunotherapy for Dogs: Still Running Behind Humans}

\author{
Hans Klingemann * \\ ImmunityBio Inc., Culver, CA, United States
}

Despite all good intentions, dogs are still running behind humans in effective cancer immunotherapies. The more effective treatments in humans, like infusions of CAR-T and NK-cells are not broadly pursued for canines due to significant costs, the rather complicated logistics and the lack of targetable surface antigens. Monoclonal antibodies are challenging to develop considering the limited knowledge about canine target antigens and about their mode of action. Although immunogenic vaccines could be less costly, this approach is hampered by the fact that cancer by itself is immunosuppressive and any preceding chemotherapy may suppress any clinically meaningful immune response. This review - rather than providing a comprehensive listing of all available immunotherapies for dogs, aims at pointing out the issues that are holding back this field but which hopefully can be addressed so that dogs can "catch up" with what is available to humans.

Keywords: dogs, immunotherapy, canine, T-cells, natural killer cells, monoclonal antibodies

\section{INTRODUCTION}

Back in 2017, I published a review on the immunotherapy options for dogs with cancer. Somewhat provocatively the paper was entitled: Immunotherapy for dogs: Running behind humans (1). The years have gone by and it is time to assess if our dogs have caught up. Looking at what has been accomplished in those 4 years, it is fair to conclude that canine immunotherapy has come a few steps closer but certainly a lot more work needs to be done.

There are several reasons why this area is moving rather slowly (Table 1). Any immunotherapy that profit-minded companies would be interested in commercializing for the canine market would have to be offered at a prize that dog owners feel comfortable paying. Since only about $10 \%$ of dog owners have health insurance for their pet, there is a ceiling what owners can and will spend on complex treatments. Especially cell-based cancer treatments (T-cells, NK-cells) require good manufacturing practices (GMP) compliant ex vivo cell expansion, special instrumentation, cytokines and trained personnel, easily accumulating costs of several thousands of dollars. Hence, companies (with some exceptions) will not be eager to develop those treatments.

To potentially alleviate this issue, "comparative oncology" has gained some popularity [reviewed in (2)]. The premise is that there are overlapping similarities/characteristics of certain cancers between humans and dogs that are also reflected at the genetic level. Hence hard to fund academic investigators may welcome this model, which is supported by NIH grants, as any positive result could potentially benefit drug development for humans. However, the concern is that the dog is becoming a better mouse model for exploring new therapeutics for humans. Also, the comparative 
TABLE 1 | Reasons why immunotherapy for dogs is still lagging behind humans

- Developing cellular immunotherapies are expensive and pharmaceutical companies have limited enthusiasm to develop them for canines assuming the projected profit is limited

- Targetable surface molecules (of the CD family) are less well characterized than in humans

- Effector mechanism for monoclonal antibodies are incompletely known: which cells can mediate ADCC and which IgG subclasses are utilized for ADCC

- Lack of reagents to characterize comprehensively dog's immune cells and cytokine profile

- Conducting randomized controlled clinical trials is challenging (breed variability, disease staging, compliance in case of progression)

oncology concept might only apply to cancers that are common in dogs and humans like lymphoma, osteosarcoma and melanoma, but certainly not for the majority of human malignancies such as cancer of the lung, colon, breast, prostate and pancreas. Although the human and the canine genome have roughly $80 \%$ homology, discovery of new immuno-therapeutics based on this homology is limited and largely restricted to small molecules. A recent study tested the cross-reactivity of seven FDA approved human immune checkpoint inhibitors (ICI) with dog immune cells and only two IPIs had (limited) crossreactivity (S. Pantelyushin, personal communication). Moreover, If given to dogs, an immune response would be expected to those human proteins. Hence there are limitations what comparative oncology can offer when it comes to testing human cell and protein based therapeutics for applications in dogs.

The following review is not about a comprehensive listing of immunotherapies that are available for dogs, being developed or may even be promising - there are excellent reviews that have accomplished just that (3-5). This article is more of an assessment of what the issues are that are holding dogs back from catching up with immunotherapy options that are available for humans.

\section{CELLULAR IMMUNOTHERAPY WITH CYTOTOXIC LYMPHOCYTES (T - CELLS AND NK -CELLS)}

The most effective cellular immunotherapies in the human space as of now - besides allogeneic stem cell transplantation - involve CAR engineered T-cells (CAR-T) and monoclonal antibodies $(m A b)(6)$. The costs for preparing a CAR T-cell product for a human patient is currently in the $\$ 350 \mathrm{~K}$ range. Even with the smaller size of a dog and somewhat less stringent GMP and regulatory issues to comply with, the costs for this treatment will still be substantial - but cost considerations are just one aspect: it still is unknown how best to generate CAR-T cells that can target canine cancer antigens - which are also insufficiently characterized. The dog equivalent of CD19 has not been identified yet and only one study looked at anti-CD20 CAR-T cells transfected with mRNA (7). As expected for mRNA transfection, the duration of CAR expression was short. It is known from human CAR-T cell studies that the T-cells have to clonally expand and persist in the recipient to show efficacy (8). Based on this short expression, no conclusions can be drawn about potential efficacy and side effects with CAR-T cells in canine patients.

At the recent SITC (Society for Immunotherapy of Cancer) meeting, the Seattle group presented data on CAR-T cell treatment in 2 dogs (9). The CAR-T cells were directed against the human B7/ $\mathrm{H} 3$ surface antigen on solid tumors which the authors showed to be cross-reactive between humans and canines. In contrast to the previous study, the T-cells were transduced retrovirally which resulted in about $70 \%$ transduction efficiency. The canine recipients also received lymphodepleting chemotherapy (Fludarabine/Cyclophosphamide) prior to infusion. Persistence of the CAR-T cells in the canine blood could be demonstrated for several weeks via a co-transfected EGFR tag. $10^{9} \mathrm{CAR}-\mathrm{T}$ cells $/ \mathrm{m}^{2}$ were infused and only mild liver enzyme abnormalities were noted.

Some preliminary in vitro results were recently reported with canine - T cells transfected with RNA for a caninenized CAR construct. This construct targeted the canine IL13a2 receptor through the human Fab portion of the CAR (the remaining CAR components were canine) (10). The target tumor for this approach would be glioma, but the issue of time limited RNA expression and any immunogenicity of the human Fab need to be addressed.

Considering the challenges with CAR-T cell therapy in dogs, alternative cellular therapies deserve consideration. ELIAS Animal Health has developed a cellular therapy for osteosarcoma that uses a vaccination step with the patient's tumor tissue followed by apheresis to harvest the dog's $\mathrm{T}$ - cells. Those are then ex vivo activated (using a proprietary formula) and expanded. The cell preparation is sent back to the veterinarian for infusion (11). An update on the study results were recently presented (Bryan, 2020, personal communication $)^{1}$, and if the data hold, this could present a treatment options for dogs with (non-metastatic) osteosarcoma and potentially for other cancers as well. A larger controlled study with carboplatinum as the control group is underway.

NK cells are currently seeing a surge in clinical trials in humans. Their advantage over T- cells is their safety profile (no cytokine storm when expressing a CAR) but their life span is shorter. For the human patient, investigators are using cord blood or peripheral blood NK cells which may require depletion of any allogeneic T-cells (12). The continuously growing NK-92 cell line (13) provides a logistically easier effector NK-cell pool and clinical trials with PD-L1 expressing NK-92 cells ( $t-h_{a N K}{ }^{\mathrm{TM}}$ ) in humans are ongoing (14). With the advent of CRISPR/Cas9 those cells can be genetically engineered such that they are no longer immunogeneic for canine T-cells. Treatment of canine osteosarcoma and histiocytoma with cells from the human clonal NK/T cell line (TALL 104) in the late 1990's had shown some encouraging anti-tumor effects $(15,16)$.

Human NK cells express the characteristic CD56 antigen and are negative for CD3 expression. In contrast, NK cells from dogs are less well defined (17). Since their first description in 2000 (18), not much additional characteristics have been identified. What has been described in the literature is that that these "NK-

\footnotetext{
${ }^{1}$ Paws for Cure Research Symposium
} 
like" cells have granular morphology, express CD5 at low density and are CD3 and CD56 negative $(19,20)$. The canine surface markers coded by the NCR1 gene p46 (21) and CD94 (22) seem to be expressed variably on these cells and their full relevance is still poorly understood.

This lack of deeper knowledge about canine NK cells has negatively affected the clinical development of NK-based immunotherapy for dogs. The team at UC Davies has infused ex vivo activated "NK-like" cells in conjunction with local radiation for treatment of osteosarcoma (23). The canine NK cells were isolated from PBMC based on CD5dim/NKp46 expression and then expanded ex vivo for two weeks on a K562 feeder layer modified with mIL21 and in the presence of recombinant human IL-2 (100 IU/mL). Those expanded NK cells were then infused after local tumor radiation. The rationale for this approach is based on their pre-clinical work showing that radiation sensitizes the tumor to the cytotoxic effects of NK cells and also directs their migration. The potential synergy between local radiation and immunotherapy has been reviewed by Thamm (24).

In humans, NK cells are also essential cytotoxic effector cells for antibody dependent cellular cytotoxicity (ADCC) by binding mAbs through the CD16 Fc $\gamma$-receptor. For dogs it has not been conclusively demonstrated that "NK-like" cells can mediate ADCC against antibody-coated tumor cells and that they express a human Fc $\gamma$-receptor/CD16 equivalent.

\section{CYTOKINES}

Although a portfolio of canine recombinant cytokines is available for laboratory research, no canine specific cytokines are commercially available for treatment of dogs (4). Human IL-2 and IL-12 (including NHS-IL-12, a fully human IgG immunocytokine) have been given to dogs with cancer - but these cytokines generally require higher doses than in humans. Except for a modest efficacy signal with NHS-IL-12 (25), convincing data on in vivo tumor control are largely missing. The same is true for the intra-tumor electro-application of a plasmid based IL-12 construct $(26,27)$.

Under the auspices of the PRECINCT Canine Trials Network the (human) IL-15 superagonist Anktiva $^{\mathrm{R}}$ is being tested in combination with inhaled (human) IL-15 in dogs with melanoma or osteosarcoma driven lung metastases [PIs: Canter RJ, Rebhun RB. [https://www.precinctnetwork.org].

\section{MONOCLONAL ANTIBODIES}

A striking example about the gap between what is available to humans versus dogs is the area of mAbs. There are no mAbs for dogs approved and effective for cancer therapy. The CD20 antibody from Arantana/Elanco is approved by the USDA (28) but has not stood the test of time for effectiveness (29).
Not unexpectedly, human mAbs generally do not cross-react with canine target cells - in the case of rituximab, the most widely used $\mathrm{mAb}$ in the treatment of human lymphoma, this can be traced back to a single amino acid difference in the active binding site (30). There is some suggestion that cetuximab (antiEGFR) and trastuzumab (anti-Her-2) can bind to some canine cancer cell lines (31). The larger issue relates to the fact that the human protein will induce an anti-human immune response in dogs (humoral and cellular). Some caninenized mAbs (only the complementary sequences are non-canine) have received conditional approval by the USDA for lymphoma (i.e. Blontress ${ }^{\circledR}$, Tactress ${ }^{\circledR}$ ). Disappointingly no peer-reviewed clinical evidence of efficacy for those mAbs has been published. Elanco's anti-CD20 mAb (1E4) has shown to deplete B-cells in healthy Beagles with an in vivo half-life of about 2 weeks, but the $\mathrm{mAb}$ is still undergoing clinical efficacy testing (32). Recently a chimeric (rat) anti-canine CD20 mAb (defucosylated) has been reported (33) which significantly depleted B-cells in the blood of beagles $(n=8)$ lasting for about 4 weeks. Clinical data are still pending. The Pittsburgh group recently described a anti-canine CD19 mAb (4E9) based on immunohistology. Data to its therapeutic relevance are not available yet (34).

For $\mathrm{mAb}$ treatment to be effective in dogs, it is important to remember how mAbs deploy their therapeutic effect: (i) neutralizing antibodies bind to the target cells and prevent those cells from further functioning/dividing; (ii) direct cytotoxic mechanisms involve complement mediated cell lysis (CMC) and (iii) antibody dependent cellular cytotoxicity (ADCC). Not much is known about the complement system in dogs. ADCC in humans is executed predominantly by natural killer (NK) cells through their Fc-receptor (CD16) that binds $\mathrm{mAbs}$ of the IgG1 or IgG3 subclass. A CD16 like receptor has not been described on canine NK cells and little is also known about which IgG subtypes in dogs can execute ADCC. The IgG subclasses in dogs are designated A,B,C,D and it appears from preliminary studies that B and D may be involved in ADCC (35). Since NK cells are rather incompletely defined in dogs, their contribution in ADCC is largely unknown although an earlier study had suggested some ADCC mediated by canine large granular lymphocytes isolated based on surface markers (CD3 - CD21- CD5- TCR $\alpha \beta-$ TCR $\gamma \delta)$, activated and expanded for about two weeks with various cytokines on a K562 feeder layer (36).

Sine only a rather limited number of canine cancer antigens are known, investigators have been looking into human mAbs that may be cross-reactive. The clinical relevance though is limited considering that those antibodies, like trastuzumab or cetuximab, mostly work through $\operatorname{ADCC}(37,38)$. In addition, the immunogenicity of these human proteins is a concern.

Stipulated by the positive outcome data with immune checkpoint inhibitors (ICIs) in some human cancers, especially melanoma and lymphoma (39), the search for canine mAb equivalents has gained some traction $(40,41)$. First reports about the distribution and function especially of PD-1 for immune-cells and its ligand PD-L1 on tumor tissues are just emerging $(42,43)$. A canine chimeric $\mathrm{mAb}$ (the Fab site being 
rat-derived) against $\mathrm{PD}$-L1 was tested in dogs with oral melanoma and sarcoma (44). All nine tumors ( $\mathrm{n}=7$ melanoma, $\mathrm{n}=2$ sarcoma) expressed PD-L1, based on immunohistology. Only two temporary partial responses after multiple infusions were seen in a dog with melanoma and in one dog with sarcoma.

A Japanese team recently presented data on two canine $P D-1$ antibodies: one being a rat-dog chimeric $\mathrm{mAb}$ and the other one is a caninenized antibody with the complementary segment being rat derived (45). Thirty dogs with oral melanoma were treated with a mean of about 8 infusions. Side effects (mostly grade 1) were seen in 19/30 dogs but with one death caused by the mAb. Four dogs with stage IV oral melanoma had an objective temporary response and some stable diseases were noted with the majority of dogs progressing on treatment. Compared to matched historical controls, overall survival was not prolonged.

Since human and canine immune checkpoint inhibitors (ICIs) have about $70-80 \%$ sequence homology, a Swiss group tested the blocking potential and functional effects of seven FDAapproved human ICIs, targeting CTLA-4 and the PD-1/PD-L1 against canine tissue (S. Pantelyushin, personal communication). Out of seven candidates only atezolizumab and to a lesser extent avelumab increased canine T-cell cytokine production in vitro. Although these in vitro data are interesting, they will unlikely stipulate trials in dogs because of the immunogenic potential of the human protein structure. Considering the available data with canine ICI, it would be reasonable to conclude that the relevance of current ICIs in dogs is rather limited especially when taking the cost/benefit ratio into account.

\section{TUMOR VACCINES AND ONCOLYTIC VIRUSES}

If proven effective, tumor vaccines could be logistically simple and provide a relative affordable immunotherapy for dogs. Different approaches are currently being considered. The vaccine is usually administered after initial treatment of the cancer when the patient is in presumed remission. Vaccines can be generated against neoepitopes on cancer cells, often against driver oncogens like mutated p53, KRAS, Her-2 applied as peptides (46). Recent data presented at the 2020 SITC meeting (47) showed in a mouse model that autologous tumor infiltrating lymphocytes (TIL) were only effective against p53 expressing tumors after the T-cells had been engineered to express an anti-p53 specific T-cell receptor. This observation is relevant with regard to a more general problem with tumor vaccination: unmodified autologous $\mathrm{T}$-cells are ineffective effector cells, having become tolerant and "exhausted" due to the underlying cancer and chemotherapy.

Another group of cancer vaccines is supposed to stimulate the dog's immune system non-specifically without providing a cancer specific antigen. There are currently two DNA based vaccines approved by the USDA: Oncept ${ }^{\circledR}$ (human DNA based) is given to dogs with oral melanoma. Unfortunately, no properly controlled studies beyond comparison with historical controls are available $(48,49)$. Essentially the same applies for a murine DNA vaccine (also from Merial/Boehringer) for canine lymphoma. Both vaccines are widely used in the veterinary practice - their reasonable costs, the fact that they are USDA approved and that the veterinarian feels that something can be offered to the dog's cancer treatment, are motivators for prescribing these vaccines.

A recombinant xenogeneic (human Her-2/neu expressing) Listeria based vaccine against osteosarcoma was reported in 2016 from the University of Pittsburgh (50). Eighteen dogs with appendicular osteosarcoma following amputation and chemotherapy received the vaccine. The median survival time of the treatment group appeared to be significantly longer than the historical controls. Unfortunately, no "Listeria only" treatment group was included in that study and no immune response parameters against the human Her-2 sequence were reported. Adverse events in that study were mild to moderate and primarily consisted of fever, lethargy, and nausea/vomiting. In 2017 the Comparative Oncology Trials Consortium at the NCI initiated a larger trial in 80 dogs to test the vaccine across various centers. The study recently closed and reporting of results is pending. However, outcome data may be inconsequential as some serious Listeria-related infections were reported in 4 dogs that were treated with the preparation outside of the study (51, 52). Elanco Inc. (Greenfield, IN) which had been granted conditional licensure from the USDA, has decided not to develop the vaccine further.

Oncolytic viruses are believed to initiate a similar immunotherapy response as vaccines in addition to providing a potential direct cytolytic effect (53). Oncolytic viruses can have selectivity for malignant cells where they replicate and - by lysing the "host" cell - expose and present the antigens to immune cells. Only limited research in this area has been done for canine cancers although some initial positive results are available in canine melanoma with an adenovirus based (human) CD40 Ligand (54).

The Vaccination Against Canine Cancer Study (VACCS) is in a special category as the vaccine is a "gemisch" of approximately 30 abnormal proteins found on the surface of common canine cancers (lymphoma, osteosarcoma, hemangiosarcoma and mastocytoma) (55). These "abnormal" proteins are the result of frameshift mutations resulting in improperly coded RNA. The vaccine is given prophylactically to dogs at high risk while they are still healthy. It remains to be seen if this approach can generate memory $\mathrm{T}$-cells that are able to react when a tumor arises that expresses the protein that was part of the initial vaccine.

\section{IMMUNE-ACTIVE SMALL MOLECULE CANDIDATES}

Genetic abnormalities of canine cancers have become the emphasis for small molecule drug development to potentially interfere with the abnormality. It remains to be seen if sequence variations in the genome are sufficiently informative. Human studies suggest that genetic changes in cancer are multiple and 
complex and that it is more relevant to look at proteomics as not all genetic abnormalities result in a tumor inducing/promoting molecule (56) Efforts are underway to apply transcriptional analysis of tumor tissue to come up with immune-active combinations that would target any alteration. As part of the Cancer Moonshot U01 grant, clinical studies are underway using such targets as: CXCR1, CCR2, VGFR/Flt3/gp130 (S. Dow, personal communication).

\section{IMMUNOLOGICAL MODULATION OF THE TUMOR MICROENVIRONMENT}

Increasing attention is focused around the relevance of the tumor microenvironment (TME) to support and maintain malignant transformation. Our knowledge about the immunological makeup of the TME is still quite sketchy largely due to the lack of reliable in vitro models. From studies with human cells it is known that T-regulatory cells (Tregs) and immunosuppressive (type II) macrophages in the TME can negatively affect the antitumor immune response (57). It was recently reported that blockade of CCR4 signaling with a humanized antibodies

\section{REFERENCES}

1. Klingemann H. Immunotherapy for Dogs: Running Behind Humans. Front Immunol (2018) 9:1-6. doi: 10.3389/fimmu.2018.00133

2. LeBlanc AM, Mazcko CN. Improving Human Cancer Therapy Through the Evaluation of Pet Dogs. Nat Rev Cancer (2020) 20:728-42. doi: 10.1038/ s41568-020-0297-3

3. Anderson K, Modiano JF. Progress in Adaptive Immunotherapy for Cancer in Companion Animals: Success on the Path to a Cure. Vet Sci (2015) 2:363-87. doi: $10.3390 /$ vetsci2040363

4. Dow S. A Role for Dogs in Advancing Cancer Immunotherapy Research. Front Immunol (2019) 10:2935. doi: 10.3389/fimmu.2019.02935

5. Thamm DH. Canine Cancer: Strategies in Experimental Therapeutics. Front Oncol (2019) 9:1257. doi: 10.3389/fonc.2019.01257

6. Kim DW, Cho J-Y. Recent Advances in Allogeneic CAR-T Cells. Biomolecules (2020) 10:263. doi: 10.3390/biom10020263

7. Panjwani MK, Smith JB, Schutsky K, Gnanandarajah, O'Connor C, Powell D Jr, Mason NJ. Feasibility and Safety of RNA-Transfected CD20-Specific Chimeric Antigen Receptor T-Cells in Dogs With Spontaneous B-Cell Lymphoma. Mol Ther (2016) 24:1602-14. doi: 10.1038/mt.2016.146

8. Mueller KT, Maude SL, Porter DL, Frey N, Wood P, Han X, et al. Cellular Kinetics of CTL019 in Relapsed/Refractory B-Cell Acute Lymphoblastic Leukemia and Chronic Lymphocytic Leukemia. Blood (2017) 130:2317-25. doi: 10.1182/blood-2017-06-786129

9. Zhang S, Kohli K, Black RG. Establishment of Canine CAR T - Cells Treatment Model for Solid Tumor Immunotherapy Development. J Immunother Cancer (2020) 8(Suppl 3):A133. doi: 10.1136/jitc-2020-SITC2020.0139

10. Yin Y, Boesteanu AC, Binder ZA, Xu C, Reid RA, Rodrigues JL, et al. Checkpoint Blockade Reverses Anergy in IL-13R 22 Humanized scFv-Based CAR T Cells to Treat Murine and Canine Gliomas. Mol Ther Oncolytics (2018) 11:20-38. doi: 10.1016/j.omto.2018.08.002

11. Flesner BK, Wood GW, Gayheart-Walsten P, Sonderegger L, Henry CJ, Tate DJ, et al. Autologous Cancer Cell Vaccination, Adoptive T-Cell Transfer, and Interleukin-2 Administration Results in Longterm Survival for Companion Dogs With Osteosarcoma. J Vet Intern Med (2020) 34:2056-67. doi: 10.1111/ jvim. 15852

12. Klingemann H. Challenges of Cancer Therapy With Natural Killer Cells. Cytotherapy (2015) 17:245-9. doi: 10.1016/j.jcyt.2014.09.007 could deplete Tregs in a canine model of invasive bladder cancer resulting in sustained tumor regression and prolonged survival (58)

\section{WHAT NEXT?}

In order to offer dogs novel immunotherapies that truly benefits them, we need more basic research that defines in more detail the components of the canine immune system and its interaction with the tumor in the context of the its microenvironment. Also, a more detailed analysis of cancer antigens, their immunogenicity and targetability need to occur. Just initiating clinical trials in dogs to explore new drugs/molecules that potentially would benefit humans without comprehensive knowledge of the specifics of the dog's immune system, will not benefit them.

\section{AUTHOR CONTRIBUTIONS}

The author confirms being the sole contributor of this work and has approved it for publication.

13. Gong JH, Maki G, Klingemann HG. Characterization of a Human Cell Line (NK-92) With Phenotypical and Functional Characteristics of Activated Natural Killer Cells. Leukemia (1994) 4:652-8.

14. Fabian KP, Padget MR, Donahue RN, Solocinsk K, Robbins Y, Allen CT, et al. PD-L1 Targeting High-Affinity NK (t-haNK) Cells Induce Direct Antitumor Effects and Target Suppressive MDSC Populations. J Immunother Cancer (2020) 8:e000450. doi: 10.1136/jitc-2019-000450

15. Cesano A, Visonneau S, Jeglum KA, Owen J, Wilkinson K, Carner K, et al. Phase I Clinical Trial With a Human Major Histocompatibility Complex nonRestricted Cytotoxic T-Cell Line (TALL-104) in Dogs With Advanced Tumors. Cancer Res (1996) 56:3021-9.

16. Visonneau S, Cesano A, Jeglum KA, Santoli D. Adjuvant Treatment of Canine Osteosarcoma With the Human Cytotoxic T-Cell Line TALL-104. Clin Cancer Res (1999) 7:1868-75.

17. Gingrich AA, Modiano JF, Canter RJ. Characterization and Potential Applications of Dog Natural Killer Cells in Cancer Immunotherapy. J Clin Med (2019) 8:1802. doi: 10.3390/jcm8111802

18. McDonough SP, Moore PF. Clinical, Hematologic, and Immunophenotypic Characterization of Canine Large Granular Lymphocytosis. Vet Pathol (2000) 37:637-46. doi: 10.1354/vp.37-6-637

19. Shin DJ, Park JY, Jang YY, Lee J-J, Lee Y-K, Shin M-G, et al. Ex Vivo Expansion of Canine Cytotoxic Large Granular Lymphocytes Exhibiting Characteristics of Natural Killer Cells. Vet Immunol Immunopathol (2013) 153:249-59. doi: 10.1016/j.vetimm.2013.03.006

20. Lee SH, Shin DJ, Kim Y, Kim C-J, Lee J-J, Yoon MS, et al. Comparison of Phenotypic and Functional Characteristics Between Canine Non-B, Non-T Natural Killer Lymphocytes and CD3(+)CD5(dim)CD21(-) Cytotoxic Large Granular Lymphocytes. Front Immunol (2018) 9:841. doi: 10.3389/fimmu.2018.00841

21. Grondahl-Rosado C, Boysen P, Johansen GM, Brun-Hansen H, Storcet AK. NCR1 Is an Activating Receptor Expressed on a Subset of Canine NK Cells. Vet Immunol Immunopathol (2016) 177:7-15. doi: 10.1016/j.vetimm. 2016.05.001

22. Graves SS, Gyurkocza B, Stone DM, Parker MH, Abrams K, Jochum C, et al. Development and Characterization of a Canine-Specific Anti-CD94 (KLRD1) Monoclonal Antibody. Vet Immunol Immunopathol (2019) 211:10-8. doi: 10.1016/j.vetimm.2019.03.005

23. Canter RJ, Grossenbacher SK, Foltz JA, Sturgill IR, Park JS, Luna JI, et al. Radiotherapy Enhances Natural Killer Cell Cytotoxicity and Localization in 
Pre-Clinical Canine Sarcomas and First-in-Dog Clinical Trial. J Immunother Cancer (2017) 5:98. doi: 10.1186/s40425-017-0305-7

24. Thamm DH. Interactions Between Radiation Therapy and Immunotherapy: The Best of Two Worlds? Vet Comp Oncol (2006) 4:189-97. doi: 10.1111/ j.1476-5829.2006.00110.x

25. Paoloni M, Mazcko C, Selting K, Lana S, Barber L, Phillips J, et al. Defining the Pharmacodynamic Profile and Therapeutic Index of NHS-IL12 Immunocytokine in Dogs With Malignant Melanoma. PloS One 10:e0129954. doi: 10.1371/ journal.pone.0129954

26. Cicchelero L, Denies S, Haers HL, Vanderperren K, Stock E, Van Brantegem, et al. Intratumoural Interleukin 12 Gene Therapy Stimulates the Immune System and Decreases Angiogenesis in Dogs With Spontaneous Cancer. Vet Comp Oncol (2016) 400:205-18. doi: 10.1111/vco.12255

27. Lampreht Tratar U, Kos S, Kamensek U. Antitumor Effect of Antibiotic Resistance Gene-Free Plasmids Encoding Interleukin-12 in Canine Melanoma Model. Cancer Gene Ther (2018) 25:260-73. doi: 10.1038/s41417-018-0014-5

28. Available at: http://aratana.investorroom.com/2015-09-24-AratanaTherapeutics-Provides-Product-Updates.

29. Niedziela K. Arantana Suffers Setback on Canine Lymphoma Products. Vet Pract News (2015)

30. Impellizeri JA, Howell K, McKeever KP, Crow SE. The Role of Rituximab in the Treatment of Canine Lymphoma: An Ex Vivo Evaluation. Vet J (2006) 171:556-8. doi: 10.1016/j.tvjl.2005.03.005

31. Singer J, Weichselbaumer M, Stockner T, Mechtcheriakova D, Sobanov Y, Bajna E, et al. Comparative Oncology: ErbB-1 and ErbB-2 Homologues in Canine Cancer are Susceptible to Cetuximab and Trastuzumab Targeting. Mol Immunol (2012) 50:200-9. doi: 10.1016/j.molimm.2012.01.002

32. Rue SM, Eckelman BP, Efe JA, Bloink K, Deveraux QL, Lowery D, et al. Identification of a Candidate Therapeutic Antibody for Treatment of Canine B-Cell Lymphoma. Vet Immunol Immunopathol (2015) 164:148-59. doi: 10.1016/j.vetimm.2015.02.004

33. Mizuno T, Kato Y, Kaneko MK, Sakai Y, Shiga T, Kato M, et al. Generation of a Canine Anti-Canine CD20 Antibody for Canine Lymphoma Treatment. Sci Rep (2020) 10:11476. doi: 10.1038/s41598-020-68470-9

34. Haran KP, Lockhart A, Xiong A, Radaelli E, Savickas PJ, Posey A, et al. Generation and Validation of an Antibody to Canine CD19 for Diagnostic and Future Therapeutic Purposes. Vet Pathol (2020) 57:241-52. doi: 10.1177/ 0300985819900352

35. Bergeron LM, McCandless EE, Durham S, Dunkle B, Zhu Y, Shelly Y, et al. Comparative Functional Characterization of Canine IgG Subclasses. Vet Immunol Immunopathol (2014) 157:31-41. doi: 10.1016/j.vetimm.2013.10.018

36. Shin DJ, Park JY, Jang YY, Lee J-J, Lee YK, Shin M-G, et al. Ex Vivo Expansion of Canine Cytotoxic Large Granular Lymphocytes Exhibiting Characteristics of Natural Killer Cells. Vet Immunol Immunopathol (2013) 153:249-59. doi: 10.1016/j.vetimm.2013.03.006

37. Kurai J, Chikumi H, Hashimoto K, Yamaguchi K, Yamasaki A, Sako T, et al. Antibody-Dependent Cellular Cytotoxicity Mediated by Cetuximab Against Lung Cancer Cell Lines. Clin Cancer Res (2007) 13:1552-61. doi: 10.1158/ 1078-0432.CCR-06-1726

38. Collins DM, O’Donovan N, McGowan PM, O'Sullivan F, Crown J. Trastuzumab Induces Antibody-Dependent Cell-Mediated Cytotoxicity (ADCC) in HER-2-non-Amplified Breast Cancer Cell Lines. Ann Oncol (2012) 7:1788-95. doi: 10.1093/annonc/mdr484

39. Murciano-Goroff YR, Warner AB, Wolchok JD. The Future of Cancer Immunotherapy: 20 Microenvironment-Targeting Combinations. Cell Res (2020) 30:507-19. doi: 10.1038/s41422-020-0337-2

40. Coy J, Caldwell A, Chow L, Guth A, Dow S. PD-1 Expression by Canine T Cells and Functional Effects of PD-1 Blockade. Vet Comp Oncol (2017) 15:1487-502. doi: 10.1111/vco.12294

41. Nemoto Y, Shosu K, Okuda M, Noguchi S, Mizuno T. Development and Characterization of Monoclonal Antibodies Against Canine PD-1 and PD-L1. Vet Immunol Immunopathol (2018) 198:19-25. doi: 10.1016/j.vetimm.2018.02.007

42. Hartley G, Elmslie R, Dow S, Guth A. Checkpoint Molecule Expression by B and T Cell Lymphomas in Dogs. Vet Comp Oncol (2018) 16:352-60. doi: 10.1111/vco.12386

43. Choi JW, Withers SS, Chang H, Spanier JA, De La Trinidad VL, Panesar H, et al. Development of Canine PD-1/PD-L1 Specific Monoclonal Antibodies and Amplification of Canine T Cell Function. PloS One (2020) 15:e235518. doi: 10.1371/journal.pone.0235518
44. Maekawa N, Konnai S, Takagi S, Kagawa Y, Okagawa T, Nishimori A, et al. A Canine Chimeric Monoclonal Antibody Targeting PD-L1 and its Clinical Efficacy in Canine Oral Malignant Melanoma or Undifferentiated Sarcoma. Sci Rep (2017) 7:8951. doi: 10.1038/s41598-017-09444-2

45. Igase M, Nemoto Y, Itamoto K, Tani K, Nakaichi M, Sakurai M, et al. A Pilot Clinical Study of the Therapeutic Antibody Against Canine PD-1 for Advanced Spontaneous Cancers in Dogs. Sci Rep (2020) 10:18311. doi: 10.1038/s41598-020-75533-4

46. Doyle HA, Koski RA, Bonafé N, Bruck RA, Tagliatela AM, Gee RJ, et al. Epidermal Growth Factor Receptor Peptide Vaccination Induces CrossReactive Immunity to Human EGFR, HER2, and HER3. Cancer Immunol Immunother (2018) 67:1559-69. doi: 10.1007/s00262-018-2218-9

47. Kim P, Malekzadeh P, Vale N, Hedges E, Zacharakis N, Rosenberg SA. Adoptive T Cell Therapy Targeting Somatic P53 Mutations. J Immunother Cancer (2020) 8(Suppl 3):A152. doi: 10.1136/jitc-2020-SITC2020.0152

48. Ottnod JM, Smedley RC, Walshaw R, Hauptman JG, Kiupel M, Obradovich JE, et al. A Retrospective Analysis of the Efficacy of Oncept Vaccine for the Adjunct Treatment of Canine Oral Malignant Melanoma. Vet Comp Oncol (2013) 11:219-29. doi: 10.1111/vco.12057

49. Verganti S, Berlato D, Blackwood L, Amores-Fuster I, Polton GA, Elders R, et al. Use of Oncept Melanoma Vaccine in 69 Canine Oral Malignant Melanomas in the UK. J Small Anim Pract (2017) 58:10-6. doi: 10.1111/jsap.12613

50. Mason NJ, Gnanandarajah JS, Engiles JB, Gray F, Laughlin D, GaurnierHausser A, et al. Immunotherapy With a HER2-Targeting Listeria Induces HER2-Specific Immunity and Demonstrates Potential Therapeutic Effects in a Phase I Trial in Canine Osteosarcoma. Clin Cancer Res (2016) 22:4380-90. doi: 10.1158/1078-0432.CCR-16-0088

51. Musser ML, Berger EP, Tripp CD, Clifford CA, Bergman PJ, Johannes CM. Safety Evaluation of the Canine Osteosarcoma Vaccine, Live Listeria Vector. Vet Comp Oncol (2020). doi: 10.1111/vco.12642

52. Musser ML, Berger EP, Parsons C, Kathariou S, Johannes CM. Vaccine Strain Listeria Monocytogenes Abscess in a Dog: A Case Report. BMC Vet Res (2019) 15:467-71. doi: 10.1186/s12917-019-2216-y

53. Sanchez D, Cesarman-Maus G, Amado-Molina A, Lizano M. Oncolytic Viruses for Canine Cancer Treatment. Cancers (2018) 10:404-28. doi: $10.3390 /$ cancers 10110404

54. Westberg S, Sadeghi A, Svensson E, Segall T, Dimopoulou M, Korsgren O, et al. Treatment Efficacy and Immune Stimulation by AdCD40L Gene Therapy of Spontaneous Canine Malignant Melanoma. J Immunother (2013) 36:350-8. doi: 10.1097/CJI.0b013e31829d8a1b

55. Available at: https://www.csuanimalcancercenter.org/vaccination-againstcanine-cancer-study/.

56. Frantzi M, Latosinska A, Mischak H. Proteomics in Drug Development: The Dawn of a New Era? Proteomics Clin Appl (2019) 2:e1800087. doi: 10.1002/ prca.201800087

57. Binnewies M, Roberts EW, Kersten K, Chan V, Fearon DF, Merad M, et al. Understanding the Tumor Immune Microenvironment (TIME) for Effective Therapy. Nat Med (2018) 5:541-50. doi: 10.1038/s41591-018-0014-x

58. Maeda S, Murakami K, Inoue A, Yonezawa T, Matsuki N. CCR4 Blockade Depletes Regulatory T Cells and Prolongs Survival in a Canine Model of Bladder Cancer. Cancer Immunol Res (2019) 7:1175-87. doi: 10.1158/23266066.CIR-18-0751

Conflict of Interest: The author is currently Chief Science Officer- Cellular at ImmunityBio. He is also co-founder of and equity holder in the company.

Publisher's Note: All claims expressed in this article are solely those of the authors and do not necessarily represent those of their affiliated organizations, or those of the publisher, the editors and the reviewers. Any product that may be evaluated in this article, or claim that may be made by its manufacturer, is not guaranteed or endorsed by the publisher.

Copyright (c) 2021 Klingemann. This is an open-access article distributed under the terms of the Creative Commons Attribution License (CC BY). The use, distribution or reproduction in other forums is permitted, provided the original author(s) and the copyright owner(s) are credited and that the original publication in this journal is cited, in accordance with accepted academic practice. No use, distribution or reproduction is permitted which does not comply with these terms. 